\title{
Bounce in GR and higher-order derivative operators
}

\author{
Gen $\mathrm{Ye}^{1 *}$ and Yun-Song Piao ${ }^{1,2 \dagger}$ \\ 1 School of Physics, University of Chinese Academy of Sciences, Beijing 100049, China and \\ 2 Institute of Theoretical Physics, Chinese Academy of Sciences, \\ P.O. Box 2735, Beijing 100190, China
}

\begin{abstract}
Recent progress seems to suggest that one must modify General Relativity (GR) to stably violate the null energy condition and avoid the cosmological singularity. However, with higher-order derivative operators of the scalar field (a subclass of the degenerate higher-order scalar-tensor theory), we show that at energies well below the Planck scale, fully stable nonsingular cosmologies can actually be implemented within GR.
\end{abstract}

PACS numbers:

\footnotetext{
* yegen14@mails.ucas.ac.cn

$\dagger$ yspiao@ucas.ac.cn
} 


\section{INTRODUCTION}

It is well-known that General Relativity (GR) suffers the singularity problem, which indicates that our understanding about gravity and the origin of the universe is incomplete $[1$, 2]. It is still an elusive task to look for an ultraviolet (UV)-complete theory to describe what happens at the "singularity". However, searching for fully stable nonsingular cosmologies with the effective field theory (EFT), which captures low energy behaviors of the complete theory, might be an alternative approach.

In spatially flat nonsingular cosmologies, the Null Energy Condition (NEC) must be violated for a period. However, it is often accompanied by (ghost, gradient) instabilities [3, 4], or singularities (strong coupling) in the perturbed action, see also Refs.[5-8]. Recently, it has been found that fully stable nonsingular cosmological solutions do exist in the EFT beyond Horndeski [9-14]. Degenerate higher-order scalar-tensor (DHOST) theory [15] actually is a rich pool for such EFTs [16]. However, it is noteworthy that in the nonsingular models built, the gravity has been no longer GR-like ${ }^{1}$.

Recently, the LIGO Scientific and Virgo Collaborations have detected the gravitational wave (GW) signals of binary black holes (BH) [17] and binary neutron star mergers [18], which opened a new window to probe the gravity physics. The results of all tests performed in Refs.[19, 20] showed perfect agreement with GR, particularly in the strong-field regime. Currently, GR is still a well-established effective theory in the low energy regime of the UV-complete theory, though it must break down around the Planck energy.

How to implement the nonsingular bounce with GR? It is well-known that the $P(\phi, X)$ theory can hardly bring a stable NEC violation. To stably realize such a violation, one may include higher-order derivative operators $(\square \phi)^{2},\left(\phi^{\mu \nu}\right)^{2} \cdots$ in the $P(\phi, X)$ theory, and set the EFT as, e.g.[21],

$$
L \sim \frac{M_{P}^{2}}{2}{ }^{(4)} R+P(\phi, X)+\mathcal{O}\left((\square \phi)^{2},\left(\phi^{\mu \nu}\right)^{2} \cdots\right) .
$$

Generally, higher-order corrections $\mathcal{O}\left((\square \phi)^{2},\left(\phi^{\mu \nu}\right)^{2} \cdots\right)$ are generated if one integrates out

\footnotetext{
${ }^{1}$ By "GR", we refer to a theory where matter is minimally coupled to the Einstein-Hilbert action, i.e:

$$
S=\int d^{4} x \sqrt{-g}\left(\frac{M_{p}^{2}}{2} R+L_{m}\left[g_{\mu \nu}\right]\right) .
$$

In particular, the scalar field is minimally coupled to the gravitational metric $g_{\mu \nu}$.
} 
the massive particles beyond the cutoff scale [22, 23]. One frequently studied case is $\mathcal{O} \sim$ $(\square \phi)^{2}$, see e.g.[24-26]. However, the corresponding EFT must beg unknown physics in the sufficiently far past, otherwise the higher-order derivative operator will show itself the Ostrogradski ghost. It's possible to include such higher-order derivative operators in the socalled DHOST theory [27, 28], see also [29], without introducing any Ostrogradski instability $[30,31]$.

Nevertheless, which operator in $\mathcal{O}\left((\square \phi)^{2},\left(\phi^{\mu \nu}\right)^{2} \cdots\right)$ is indispensable for achieving a pathology-free bounce in GR is still not clear so far. In this paper, we will propose a consistent (1)-like EFT for spatially-flat fully stable nonsingular cosmologies. We, with it, will discuss how to evade the No-go Theorem [3, 4] plaguing the cosmologists, and show a concrete example for the cosmological bounce.

\section{DHOST THEORY WITH $c_{T}=1$}

\section{A. Reducing to GR}

We begin with the DHOST theory with $c_{T}=1\left(c_{T}\right.$ is the speed of GWs) [32]

$$
\begin{aligned}
L_{c_{T}=1}^{D H O S T}= & P+Q \square \phi+A^{(4)} R+\frac{1}{A}\left(6 A_{X}^{2}-\left(A-X A_{X}\right) B-\frac{X^{2} B^{2}}{8}\right) \phi^{\mu} \phi_{\mu \nu} \phi_{\lambda} \phi^{\lambda \nu} \\
& +B \phi^{\mu} \phi^{\nu} \phi_{\mu \nu} \square \phi+\frac{B}{A}\left(2 A_{X}+\frac{X B}{2}\right)\left(\phi_{\mu} \phi^{\mu \nu} \phi_{\nu}\right)^{2},
\end{aligned}
$$

where $\phi_{\mu} \equiv \nabla_{\mu} \phi, \phi_{\mu \nu} \equiv \nabla_{\nu} \nabla_{\mu} \phi$ and $X \equiv \phi_{\mu} \phi^{\mu}$. The coefficients $A, Q$ and $B$ only depend on $\phi$ and $X$. According to the classification in Ref.[33], theory (2) belongs to class Ia DHOST theories. Generally, $B$ and $A$ are independent functions. However, if $B=-\frac{4}{X} A_{X}, L_{c_{T}=1}^{D H O S T}$ will reduce to the $c_{T}=1$ beyond-Horndeski theory $L_{c_{T}=1}^{b H}[34]$.

It is significant to notice that if setting $A=$ const. and $Q=0, L_{c_{T}=1}^{b H}$ will reduce to GR, while $L_{c_{T}=1}^{D H S O T}$ will become GR plus extra DHOST operators (higher-order derivative operators). The latter is not covered by the beyond-Horndeski Lagrangian [29] but belongs to a subclass of the $c_{T}=1$ DHOST theory. Degenerate conditions required by the DHOST theory guarantee that such a combination of higher-order derivative operators is free of the Ostrodradsky ghost. A (1)-like EFT will be Ostrodradsky ghost-free, only if the degenerate conditions are satisfied. 


\section{B. Perturbation in DHOST theories with $c_{T}=1$}

We adopt the ADM metric,

$$
d s^{2}=-N^{2} d t^{2}+h_{i j}\left(d x^{i}+N^{i} d t\right)\left(d x^{j}+N^{j} d t\right)
$$

where $N$ is the lapse, $N^{i}$ is the shift and $h_{i j}$ is the spatial metric. In the following we will work in the unitary gauge and use $\eta=\phi$ as the time coordinate (assuming $\phi_{\mu}$ is timelike). In particular, in this gauge $\phi_{\mu}=\delta_{\mu}^{0}$ and the dynamics of $\phi$ is absorbed into $N(\eta)$, as $\dot{\phi} \equiv d \phi / d \eta=1$ (for any operator $\mathcal{O}, \dot{\mathcal{O}}$ refers to derivatives with respect to the clock time $\phi$, or equivalently $\eta$, and not as usual to the cosmic time $t$ ).

Defining

$$
B=-\frac{4}{X} A_{X}+A \tilde{B}
$$

we have

$$
L_{c_{T}=1}^{D H O S T}=L_{c_{T}=1}^{b H}+\Delta L
$$

where

$$
\begin{aligned}
\Delta L= & A \tilde{B} \phi^{\mu} \phi^{\nu} \phi_{\mu \nu} \square \phi+\left(-A \tilde{B}+2 X A_{X} \tilde{B}-\frac{X^{2} A \tilde{B}^{2}}{8}\right) \phi^{\mu} \phi_{\mu \nu} \phi_{\lambda} \phi^{\lambda \nu} \\
& +\frac{X \tilde{B}}{2}\left(-\frac{4}{X} A_{X}+A \tilde{B}\right)\left(\phi_{\mu} \phi^{\mu \nu} \phi_{\nu}\right)^{2} .
\end{aligned}
$$

In the unitary gauge, one has $L_{c_{T}=1}^{b H}=\tilde{P}+\tilde{Q} K+A\left(\mathcal{R}-\mathcal{K}_{2}\right)[29]$, where $\mathcal{R} \equiv h^{i j} \mathcal{R}_{i j}$ is the Ricci scalar on the spacelike hypersurface, $K \equiv h^{i j} K_{i j}$ is the extrinsic curvature and $\mathcal{K}_{2} \equiv K^{2}-K_{i j} K^{i j}$. The DHOST operators follow

$$
\begin{aligned}
\phi^{\mu} \phi^{\nu} \phi_{\mu \nu} & =-\frac{1}{N^{5}}\left(\dot{N}-N^{i} \partial_{i} N\right) \equiv-\frac{1}{N^{5}} N^{\prime}, \\
\square \phi & =\frac{1}{N^{3}}\left(\dot{N}-N^{i} \partial_{i} N\right)-\frac{1}{N} K \equiv \frac{1}{N^{3}} N^{\prime}-\frac{1}{N} K, \\
\phi^{\mu} \phi_{\mu \nu} \phi_{\lambda} \phi^{\lambda \nu} & =-\frac{1}{N^{8}}\left(\dot{N}-N^{i} \partial_{i} N\right)^{2}+\frac{1}{N^{6}}(\partial N)^{2} \equiv-\frac{1}{N^{8}} N^{\prime 2}+\frac{1}{N^{6}}(\partial N)^{2} .
\end{aligned}
$$

Thus we have

$$
\Delta L=-\frac{3 A \tilde{B}^{2}}{8 N^{12}} N^{\prime 2}+\frac{A \tilde{B}}{N^{6}} N^{\prime} K-\frac{\tilde{B}}{N^{5}}\left(\frac{A}{N}+N A_{N}+\frac{A \tilde{B}}{8 N^{5}}\right)(\partial N)^{2}
$$

where the equality $X=-1 / N^{2}$ is used. Replacing $-\tilde{B} /\left(2 N^{5}\right)$ with $\tilde{B}$, we get the ADM 
form of $L_{c_{T}=1}^{D H O S T}(2)$

$$
\begin{aligned}
& L_{c_{T}=1}^{D H O S T}= \tilde{P} \\
&+\tilde{Q} K+A\left(\mathcal{R}-\mathcal{K}_{2}\right)-\frac{3 A \tilde{B}^{2}}{2 N^{2}} N^{\prime 2}-\frac{2 A \tilde{B}}{N} N^{\prime} K \\
&+\tilde{B}\left(2 \frac{A}{N}+2 A_{N}-\frac{A \tilde{B}}{2}\right)(\partial N)^{2} .
\end{aligned}
$$

We will work with (6). To study the stability of perturbations, we expand $L_{c_{T}=1}^{D H O S T}$ in (6) to second order. Defining the metric perturbation

$$
N^{i}=\delta^{i j} \partial_{j} \psi, \quad h_{i j}=a^{2}(\eta) e^{2 \zeta} \delta_{i j},
$$

we have $\mathcal{L}^{(2)}=3 a^{3} N \zeta \delta L+a^{3} \delta N \delta L+a^{3} N \delta_{2} L$ at quadratic order, where $\delta_{2} L$ refers to the expansion of $L$ at second order. To proceed, we first expand $K_{i}{ }^{j}$ and $\mathcal{R}$,

$$
\begin{gathered}
K_{i}{ }^{j}=\frac{1}{N}\left[\left(\mathcal{H}+\dot{\zeta}-\mathcal{H} \frac{\delta N}{N}\right) \delta_{i}^{j}-\frac{\delta^{j k}}{a^{2}} \partial_{i} \partial_{k} \psi\right]+\mathcal{O}\left(\delta N^{2}\right), \\
\mathcal{R}=-\frac{2}{a^{2}}\left[2 \partial^{2} \zeta+(\partial \zeta)^{2}-4 \zeta \partial^{2} \zeta\right]+\mathcal{O}\left(\zeta^{3}\right)
\end{gathered}
$$

where $\mathcal{H} \equiv \frac{d a}{a d \eta}=N H$, and $H$ is the Hubble parameter. The kinetic term in $L_{c_{T}=1}^{D H O S T}$ (6) is contributed by $-A \mathcal{K}_{2}-\frac{3 A \tilde{B}^{2}}{2 N^{2}} N^{\prime 2}-\frac{2 A \tilde{B}}{N} N^{\prime} K$. Considering (7) and (8), one finds that

$$
\mathcal{L}_{\text {kinetic }}^{(2)}=a^{3} \frac{A}{N}\left(-6 \dot{\zeta}^{2}-6 \tilde{B} \dot{\zeta} \delta \dot{N}-\frac{3 \tilde{B}^{2}}{2} \delta \dot{N}^{2}\right)=-\frac{6 a^{3} A}{N}(\dot{\zeta}+\tilde{B} \delta \dot{N} / 2)^{2}
$$

is diagonal for $\tilde{\zeta}=\zeta+\tilde{B} \delta N / 2$. The coefficients of the operators $N^{\prime 2}$ and $N^{\prime} K$ should satisfy a relation in the DHOST theory $\left(\beta_{2}=-6 \beta_{1}^{2}\right.$, see e.g.Ref.[28]). As a result, $\mathcal{L}_{\text {kinetic }}^{(2)}$ is necessarily diagonal. Confronting $\tilde{\zeta}$ with the constraint $\delta L / \delta\left(\partial^{2} \psi\right)=0$, we get

$$
\mathcal{L}^{(2)}=a^{3} N A\left[U \dot{\tilde{\zeta}}^{2}-V \frac{(\partial \tilde{\zeta})^{2}}{a^{2}}\right]
$$

with

$$
\begin{gathered}
U=\frac{\Sigma}{\gamma^{2}}+\frac{6}{N^{2}}, \\
V=\frac{2}{a A} \frac{d}{d \eta}(a \mathcal{M})-2,
\end{gathered}
$$

where

$$
\gamma \equiv\left(\frac{1}{N}+N \alpha_{B}\right) \mathcal{H}+\dot{\tilde{B}} / 2
$$




$$
\begin{gathered}
\Sigma \equiv \mathcal{H}^{2}\left[\alpha_{K}+6\left(\alpha_{B}^{2}-\frac{\gamma^{2}}{\mathcal{H}^{2} N^{2}}\right)+\frac{9 \alpha_{B} \tilde{B}}{N}+\frac{3 d\left(\alpha_{B} \mathcal{H} A \tilde{B}\right) / d \eta}{\mathcal{H}^{2} N A}\right] \\
\mathcal{M} \equiv \frac{1}{\gamma}\left[\left(\frac{A}{N}+A_{N}\right)-A \tilde{B} / 2\right]
\end{gathered}
$$

Following the notation in [28], one sets $\alpha_{B}$ and $\alpha_{K}$ as the coefficients of the operators $\delta K \delta N$ and $\delta N^{2}$ respectively,

$$
\alpha_{B}=\frac{1}{4 N A \mathcal{H}}\left(N L_{N K}+2 \mathcal{H} L_{N S}\right), \quad \alpha_{K}=\frac{1}{N A \mathcal{H}^{2}}\left(L_{N}+\frac{N}{2} L_{N N}\right),
$$

where $\mathcal{S} \equiv K_{i j} K^{i j}$. It can be checked $^{2}$ that our calculation conform with Ref.[28] by setting $N=1$.

\section{BOUNCE IN GR}

\section{A. Expelling No-go with higher-order derivative operators}

In the Horndeski theory, fully stable nonsingular cosmological solutions are prohibited, the so-called No-go Theorem [3, 4], see also [35-38] for relevant studies. One way out is going beyond Horndeski, as pointed out in Refs.[9, 10]. In particular, in the beyond-Horndeski subclass of the DHOST theory, solutions of fully stable nonsingular cosmologies have been found $[12-14,16]$.

Setting $A=M_{P}^{2} / 2=$ const. in (6), we have

$$
\begin{aligned}
& L_{c_{T}=1, A=M_{P}^{2} / 2}^{D H O S T}= \tilde{P} \\
&+\tilde{Q} K+\frac{M_{P}^{2}}{2}\left(\mathcal{R}-\mathcal{K}_{2}\right)-\frac{3 M_{P}^{2} \tilde{B}^{2}}{4 N^{2}} N^{\prime 2}-\frac{M_{P}^{2} \tilde{B}}{N} N^{\prime} K \\
&+\tilde{B}\left(\frac{M_{P}^{2}}{N}-\frac{M_{P}^{2} \tilde{B}}{4}\right)(\partial N)^{2},
\end{aligned}
$$

which also belongs to a subclass of the DHOST theory. Recall the redefinition (4) and replacement $-\tilde{B} /\left(2 N^{5}\right) \rightarrow \tilde{B}$ in Sect.II, then the coefficient $B$ in the covariant theory (2) is related to the $\tilde{B}$ in (16) by $B=-M_{P}^{2} N^{5} \tilde{B}$. It is also noticed that if $A=$ const., $\tilde{Q}=0$ in (16) is equivalent to $Q=0$ and $P=\tilde{P}$ in $L_{c_{T}=1}^{D H O S T}(2)$. Thus if $\tilde{Q}=0$, (16) is actually a (1)-like EFT.

\footnotetext{
${ }^{2}$ In Ref.[28], a different time parametrization is chosen such that $\bar{N}=1$.
} 
The essence of the No-go proof is rewriting $V>0\left(c_{S}^{2}>0\right)$ in (11) as the integral inequality, see [39] for a review,

$$
\left.a \mathcal{M}\right|_{f}-\left.a \mathcal{M}\right|_{i}>\int_{i}^{f} a A d \eta
$$

In the nonsingular models, the integral $\int_{i}^{f} a A d \eta$ will diverge, thus $\mathcal{M}$ must cross 0 at a certain time. According to (14), we have

$$
\mathcal{M}=\frac{M_{P}^{2}}{2 \gamma}\left(\frac{1}{N}-\tilde{B} / 2\right)
$$

for (16). Thus we might get $\mathcal{M}=0$ by adjusting $\tilde{B}(N, \eta)$, or equivalently $B(X, \phi)$ in $L_{c_{T}=1}^{D H O S T}$ (2). This suggests that it is possible to build fully stable nonsingular cosmological models with (16) (equivalently, (1)-like EFTs).

\section{B. An example}

To show that the observation made in Sect.III A is correct, we will present a concrete model for the nonsingular bounce, which might have significant applications in early universe scenarios, e.g.[40-43].

We adopt

$$
\mathcal{H}=H / N=\frac{\eta}{3\left(1+\eta^{2}\right)},
$$

with $N(\eta)=1$ as the background solution. When $\eta<0$, the universe contracted with $\mathcal{H} \sim 1 / \eta<0$. Cosmological bounce happened at $\eta=0$. We might set $\tilde{P}(N, \eta)$ and $\tilde{B}(N, \eta)$ in $L_{c_{T}=1, A=\text { const. }}^{\text {DHOST }}(16)$ as

$$
\tilde{P}(N, \eta)=\frac{g_{1}(\eta)}{2 N^{2}}+\frac{g_{2}(\eta)}{N^{4}}+g_{3}(\eta)
$$

and $\tilde{B}(N, \eta)=g_{4}(\eta)$. Here, since $\tilde{Q}=0, \tilde{P}(N, \eta)$ is actually equivalent to $P(X, \phi)=$ $g_{1}(\phi) X / 2+g_{2}(\phi) X^{2}+g_{3}(\phi)$ in $L_{c_{T}=1}^{\text {DHOST }}(2)$.

One simple possibility for (13) is, see also [16],

$$
\Sigma=c_{1}(\eta) \gamma^{2}
$$

According to Eq.(10), we will have $U>0$ for a suitable $c_{1}(\eta)$. Combining Eq.(21) with the background equations (A1) and (A2) in Appendix A, we get the algebraical solutions of $g_{1}(\eta), g_{2}(\eta)$ and $g_{3}(\eta)$, see Appendix B. 
Inserting $\tilde{B}(N, \eta)=g_{4}(\eta)$ into Eq.(12), we have $\gamma=\mathcal{H}+\dot{g}_{4} / 2$. Thus

$$
\mathcal{M}=\frac{M_{P}^{2}\left(1-g_{4} / 2\right)}{\dot{g}_{4}+2 \mathcal{H}} .
$$

Requiring that around $\eta=0,1-g_{4} / 2=0$ and $\dot{g}_{4} \sim \mathcal{H}($ so $\mathcal{M}=0)$, we consider such a $g_{4}$,

$$
g_{4}(\eta)=\int_{\eta}^{+\infty} 2 \mu \mathcal{H}(s) e^{-s^{2} / \lambda^{2}} d s,
$$

with $\lambda$ set by $g_{4}(0)=\mu e^{1 / \lambda^{2}} \Gamma\left(0,1 / \lambda^{2}\right) / 3=2$. Fig.1 plots the evolutions of $\dot{g}_{4}$ for $\mu=0.9$ and $\mathcal{H}$. When $|\eta| \gg \lambda, g_{4}=0$, we will have a $P(X, \phi)$ EFT with GR. Inserting (22) into (11), we have $V(\eta=0)=\frac{2(2 \mu-1)}{-\mu+1}$, so $c_{S}^{2}(\eta=0)=V / U>0$ suggests $0.5<\mu<1$.

As a concrete example, we plot Figs. 2 and 3 with $c_{1}(\eta)=150 e^{-\eta^{2} / 500}$. We see that the model is fully stable. As pointed out in Ref.[33], class Ia DHOST theories can be disformally transformed to Horndeski. It's proved in Appendix C that such field redefinition is ill-defined in the example considered here.

\section{DISCUSSION}

Currently, GR is the well-tested effective theory of gravity. Based on the higher-order derivative operators, which might capture the physics of a UV-complete theory, we propose a consistent EFT

$$
\begin{aligned}
L= & \frac{M_{P}^{2}}{2}{ }^{(4)} R+P(\phi, X)-\left(B+\frac{X^{2} B^{2}}{4 M_{P}^{2}}\right) \phi^{\mu} \phi_{\mu \nu} \phi_{\lambda} \phi^{\lambda \nu}+B \phi^{\mu} \phi^{\nu} \phi_{\mu \nu} \square \phi \\
& +\frac{X B^{2}}{M_{P}^{2}}\left(\phi_{\mu} \phi^{\mu \nu} \phi_{\nu}\right)^{2},
\end{aligned}
$$

for the spatially-flat fully stable nonsingular cosmologies. It belongs to a subclass $(A=$ $\left.M_{P}^{2} / 2, Q=0\right)$ of the $c_{T}=1$ DHOST theory (2). It has been speculated that the higherorder derivative operators $\mathcal{O}\left((\square \phi)^{2},\left(\phi^{\mu \nu}\right)^{2} \cdots\right)$ in the EFT (1) might play crucial roles in nonsingular cosmologies. Here, we clearly showed what kind of $\mathcal{O}\left((\square \phi)^{2},\left(\phi^{\mu \nu}\right)^{2} \cdots\right)$ is required for the full stability of nonsingular cosmologies.

We discussed how to evade the No-go Theorem with the EFT (24) (its ADM Langrangian (16)). In Refs.[9, 10, 12, 13], the operator $\mathcal{R} \delta g^{00}$ is used to expel the No-go. However, in their implementation, besides higher-order derivative operators, the corresponding covariant EFT also includes the derivative coupling of $\phi$ to gravity $\sim X^{(4)} R$. Here, we found 


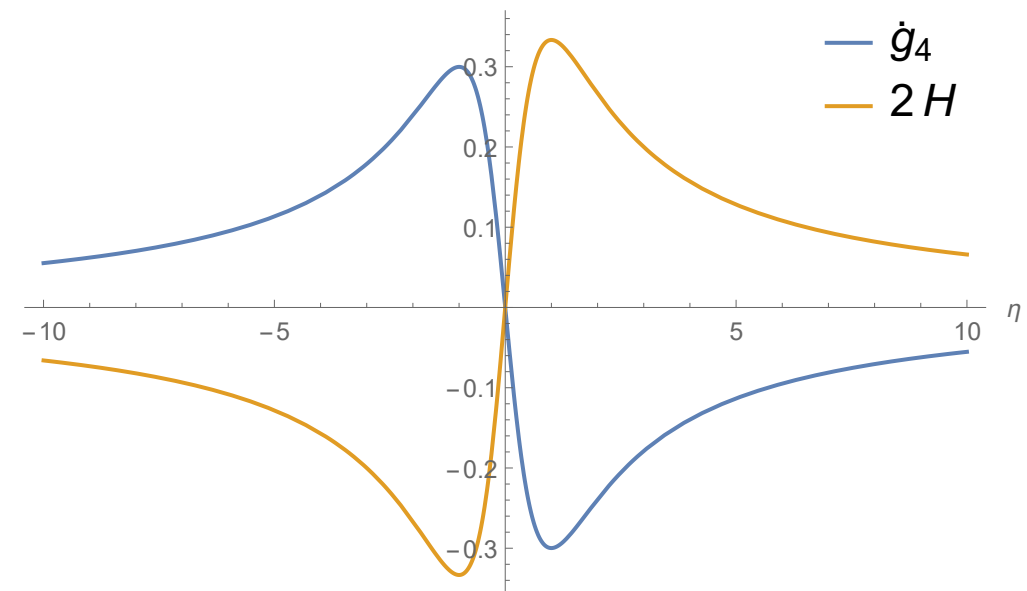

FIG. 1: $\dot{g}_{4}$ is given by (23). We require $\dot{g}_{4} \sim H$ for simplicity. We have set $\mu=0.9$ and $M_{P}=10$ in the plot.

that the No-go can be evaded solely by introducing the higher-order derivative operators $\mathcal{O}\left((\square \phi)^{2},\left(\phi^{\mu \nu}\right)^{2} \cdots\right)$ (the DHOST operators) in (24) without modifying GR. A concrete model of the cosmological bounce have been presented in Sect.III B. Generally, all the operators compatible with the symmetry of the problem are expected to be generated at quantum level. However, only a finite subset of all possible higher-order derivative operators is considered in the example studied. It would thus be interesting to study whether such model is protected against quantum corrections [44, 45]. It might be also interesting to apply the EFT (24) to regulate the singularity of the BH, e.g.[46-48].

Recently, the well-posedness of the initial value problem (IVP) has been promoted in non-perturbative cosmologies [49]. An issue worthy of exploring is whether the IVP for (24) is well-posed.

\section{Acknowledgments}

We thank Yong Cai for helpful discussions. This work is supported by NSFC, Nos.11575188, 11690021. 


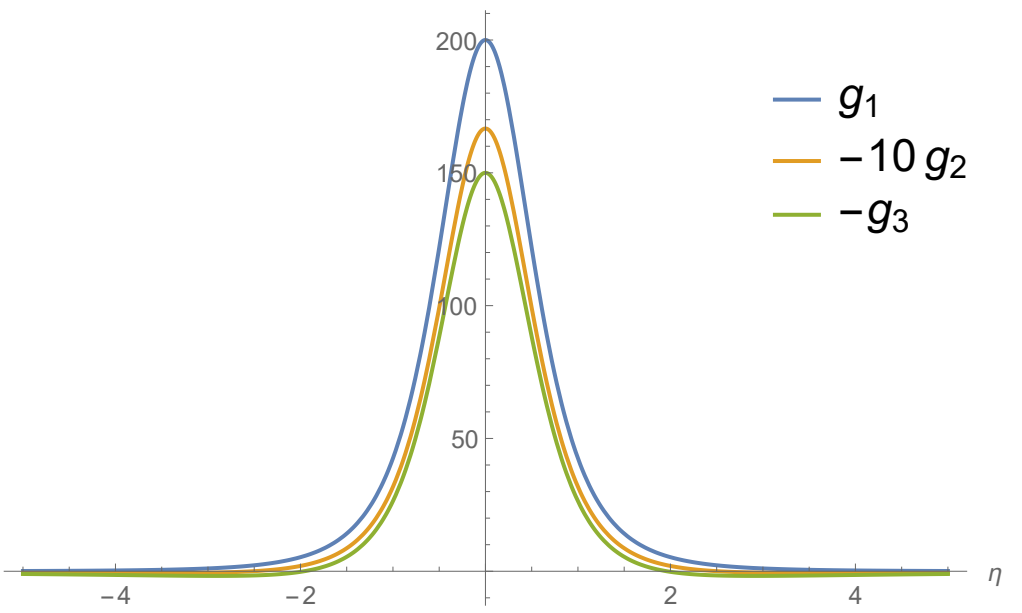

FIG. 2: Coefficients $g_{1}, g_{2}$ and $g_{3}$ in $\tilde{P}(N, \eta)(20)$.

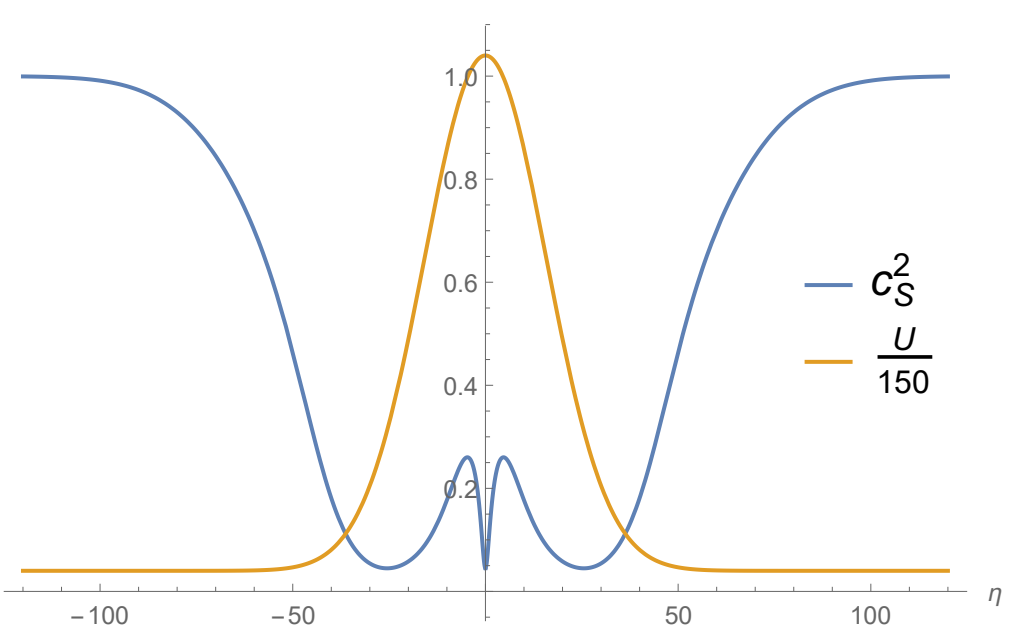

FIG. 3: Throughout the whole evolution, $c_{S}^{2}>0$, while $c_{S}^{2} \rightarrow 1$ as $|\eta| \rightarrow \infty$.

\section{Appendix A: The background equations}

Varying (6) with respect to $N$ and $\mathcal{H}$, respectively, we get

$$
\begin{aligned}
3 B\left(\frac{A B \ddot{N}}{N}+\frac{2 A \dot{\mathcal{H}}}{N}\right)= & \dot{N}^{2}\left(\frac{3 A B^{2}}{2 N^{2}}-\frac{3 A B B_{N}}{N}-\frac{3 A_{N} B^{2}}{2 N}\right)+\mathcal{H}^{2}\left(-\frac{18 A B}{N}-\frac{6 A}{N^{2}}+\frac{6 A_{N}}{N}\right) \\
& +\dot{N}\left(-\frac{9 A B^{2} \mathcal{H}}{N}-\frac{6 A B B_{\eta}}{N}-\frac{3 A_{\eta} B^{2}}{N}\right) \\
& +\mathcal{H}\left(-\frac{6 A B_{\eta}}{N}-\frac{6 A_{\eta} B}{N}-3 Q_{N}\right)-P_{N} N-P,
\end{aligned}
$$




$$
\begin{aligned}
6\left(\frac{A B \ddot{N}}{N}+\frac{2 A \dot{\mathcal{H}}}{N}\right)= & \dot{N}\left(\frac{12 A \mathcal{H}}{N^{2}}-\frac{12 A_{N} \mathcal{H}}{N}-\frac{6 A B_{\eta}}{N}-\frac{6 A_{\eta} B}{N}+3 Q_{N}\right) \\
& +\dot{N}^{2}\left(\frac{9 A B^{2}}{2 N}+\frac{6 A B}{N^{2}}-\frac{6 A B_{N}}{N}-\frac{6 A_{N} B}{N}\right) \\
& -\frac{18 A \mathcal{H}^{2}}{N}-\frac{12 A_{\eta} \mathcal{H}}{N}-3 P N+3 Q_{\eta} .
\end{aligned}
$$

Appendix B: Solutions of $g_{1}, g_{2}$ and $g_{3}$

$$
\begin{aligned}
& g_{1}=-\frac{1}{8 N}\left(4 c_{1} \mathcal{H} M_{p}^{2} N^{4} \dot{g}_{4}+2 c_{1} M_{p}^{2} g_{4} N^{4} \dot{g}_{4} \dot{N}+c_{1} M_{p}^{2} N^{5} \dot{g}_{4}^{2}+4 c_{1} \mathcal{H} M_{p}^{2} g_{4} N^{3} \dot{N}+c_{1} M_{p}^{2} g_{4}^{2} N^{3} \dot{N}^{2}\right. \\
& +4 c_{1} \mathcal{H}^{2} M_{p}^{2} N^{3}-12 \mathcal{H} M_{p}^{2} N^{2} \dot{g}_{4}-36 M_{p}^{2} g_{4} N^{2} \dot{g}_{4} \dot{N}+12 M_{p}^{2} N \dot{g}_{4} \dot{N}+6 M_{p}^{2} N^{3} \dot{g}_{4}^{2} \\
& -108 \mathcal{H}^{2} M_{p}^{2} g_{4} N^{2}-36 M_{p}^{2} g_{4} \dot{\mathcal{H}} N^{2}-72 \mathcal{H} M_{p}^{2} g_{4}^{2} N^{2} \dot{N}+24 \mathcal{H} M_{p}^{2} g_{4} N \dot{N} \\
& -24 M_{p}^{2} g_{4}^{2} N^{2} \ddot{N}+12 M_{p}^{2} g_{4} N \ddot{N}+18 M_{p}^{2} g_{4}^{2} N \dot{N}^{2}-12 M_{p}^{2} g_{4} \dot{N}^{2}+24 \mathcal{H}^{2} M_{p}^{2} N \\
& \left.+24 M_{p}^{2} \dot{\mathcal{H}} N-24 \mathcal{H} M_{p}^{2} \dot{N}\right) \\
& g_{2}=\frac{1}{32}\left(4 c_{1} \mathcal{H} M_{p}^{2} N^{5} \dot{g}_{4}+2 c_{1} M_{p}^{2} g_{4} N^{5} \dot{g}_{4} \dot{N}+c_{1} M_{p}^{2} N^{6} \dot{g}_{4}^{2}+4 c_{1} \mathcal{H} M_{p}^{2} g_{4} N^{4} \dot{N}+c_{1} M_{p}^{2} g_{4}^{2} N^{4} \dot{N}^{2}\right. \\
& +4 c_{1} \mathcal{H}^{2} M_{p}^{2} N^{4}+12 \mathcal{H} M_{p}^{2} N^{3} \dot{g}_{4}-12 M_{p}^{2} g_{4} N^{3} \dot{g}_{4} \dot{N}+4 M_{p}^{2} N^{2} \dot{g}_{4} \dot{N}+6 M_{p}^{2} N^{4} \dot{g}_{4}^{2} \\
& -36 \mathcal{H}^{2} M_{p}^{2} g_{4} N^{3}-12 M_{p}^{2} g_{4} \dot{\mathcal{H}} N^{3}-36 \mathcal{H} M_{p}^{2} g_{4}^{2} N^{3} \dot{N}+24 \mathcal{H} M_{p}^{2} g_{4} N^{2} \dot{N} \\
& -12 M_{p}^{2} g_{4}^{2} N^{3} \ddot{N}+4 M_{p}^{2} g_{4} N^{2} \ddot{N}+18 M_{p}^{2} g_{4}^{2} N^{2} \dot{N}^{2}-4 M_{p}^{2} g_{4} N \dot{N}^{2}+24 \mathcal{H}^{2} M_{p}^{2} N^{2} \\
& \left.+8 M_{p}^{2} \dot{\mathcal{H}} N^{2}-8 \mathcal{H} M_{p}^{2} N \dot{N}\right) \\
& g_{3}=-\frac{1}{32 N^{3}}\left(-4 c_{1} \mathcal{H} M_{p}^{2} N^{4} \dot{g}_{4}-2 c_{1} M_{p}^{2} g_{4} N^{4} \dot{g}_{4} \dot{N}-c_{1} M_{p}^{2} N^{5} \dot{g}_{4}^{2}-4 c_{1} \mathcal{H} M_{p}^{2} g_{4} N^{3} \dot{N}\right. \\
& -c_{1} M_{p}^{2} g_{4}^{2} N^{3} \dot{N}^{2}-4 c_{1} \mathcal{H}^{2} M_{p}^{2} N^{3}+36 \mathcal{H} M_{p}^{2} N^{2} \dot{g}_{4}+60 M_{p}^{2} g_{4} N^{2} \dot{g}_{4} \dot{N} \\
& +12 M_{p}^{2} N \dot{g}_{4} \dot{N}-6 M_{p}^{2} N^{3} \dot{g}_{4}^{2}+180 \mathcal{H}^{2} M_{p}^{2} g_{4} N^{2}+60 M_{p}^{2} g_{4} \dot{\mathcal{H}} N^{2} \\
& +108 \mathcal{H} M_{p}^{2} g_{4}^{2} N^{2} \dot{N}-24 \mathcal{H} M_{p}^{2} g_{4} N \dot{N}+36 M_{p}^{2} g_{4}^{2} N^{2} \ddot{N}+12 M_{p}^{2} g_{4} N \ddot{N} \\
& \left.-42 M_{p}^{2} g_{4}^{2} N \dot{N}^{2}-12 M_{p}^{2} g_{4} \dot{N}^{2}+72 \mathcal{H}^{2} M_{p}^{2} N+24 M_{p}^{2} \dot{\mathcal{H}} N-24 \mathcal{H} M_{p}^{2} \dot{N}\right) .
\end{aligned}
$$

In Sect.III B, since $N=1$, (B1), (B2) and (B3) will be simplified. 


\section{Appendix C: Disformal transformations}

In this appendix, we will show that the field redefinition relating the example in Sect.III B to a Horndeski theory is ill-defined. According to Ref.[33], theory (2) can be disformally transformed to a Horndeski theory by the field redefinition $\tilde{g}_{\mu \nu}=\Omega(X, \phi) g_{\mu \nu}+\Gamma(X, \phi) \phi_{\mu} \phi_{\nu}$ where

$$
\frac{\Omega_{X}}{\Omega}=\frac{4 A_{X}+B X}{4 A}, \quad \Gamma_{X}=\frac{2 A_{X} \Omega-2 A \Omega_{X}}{A X} .
$$

A necessary condition for an invertible disformal transformation is [28]

$$
\Omega-X \Omega_{X}-X^{2} \Gamma_{X} \neq 0 .
$$

For the specific example studied in Sect.III B, $A=M_{p}^{2} / 2, B=-M_{p}^{2} N^{5} g_{4}, X=-1 / \bar{N}^{2}=$ -1 , thus $\Omega-X \Omega_{X}-X^{2} \Gamma_{X}=\left(1-g_{4} / 2\right) \Omega$. According to (23), the disformal transformation is singular at the bounce point.

[1] S. W. Hawking and R. Penrose, Proc. Roy. Soc. Lond. A 314, 529 (1970).

[2] A. Borde, A. H. Guth and A. Vilenkin, Phys. Rev. Lett. 90, 151301 (2003) [gr-qc/0110012].

[3] M. Libanov, S. Mironov and V. Rubakov, JCAP 1608 (2016) no.08, 037 [arXiv:1605.05992 [hep-th]].

[4] T. Kobayashi, Phys. Rev. D 94 (2016) no.4, 043511 [arXiv:1606.05831 [hep-th]].

[5] D. A. Easson, I. Sawicki and A. Vikman, JCAP 1111, 021 (2011) doi:10.1088/14757516/2011/11/021 [arXiv:1109.1047 [hep-th]].

[6] A. Ijjas and P. J. Steinhardt, Phys. Rev. Lett. 117, no. 12, 121304 (2016) [arXiv:1606.08880 $[\mathrm{gr}-\mathrm{qc}]]$.

[7] Anna Ijjas, Paul J. Steinhardt, Phys. Lett. B 764 (2017) pp. 289-294, [arXiv:1609.01253 [gr-qc]]

[8] D. A. Dobre, A. V. Frolov, J. T. G. Ghersi, S. Ramazanov and A. Vikman, JCAP 1803 (2018) 020, [arXiv:1712.10272 [gr-qc]].

[9] Y. Cai, Y. Wan, H. G. Li, T. Qiu and Y. S. Piao, JHEP 1701, 090 (2017) [arXiv:1610.03400 $[\mathrm{gr}-\mathrm{qc}]]$,

[10] P. Creminelli, D. Pirtskhalava, L. Santoni and E. Trincherini, JCAP 1611, no. 11, 047 (2016) [arXiv:1610.04207 [hep-th]]. 
[11] Y. Cai, H. G. Li, T. Qiu and Y. S. Piao, Eur. Phys. J. C 77, no. 6, 369 (2017) [arXiv:1701.04330 [gr-qc]].

[12] Y. Cai and Y. S. Piao, JHEP 1709, 027 (2017) [arXiv:1705.03401 [gr-qc]].

[13] R. Kolevatov, S. Mironov, N. Sukhov and V. Volkova, JCAP 1708, no. 08, 038 (2017) [arXiv:1705.06626 [hep-th]].

[14] S. Mironov, V. Rubakov and V. Volkova, JCAP 1810, no. 10, 050 (2018) [arXiv:1807.08361 [hep-th]].

[15] D. Langlois, arXiv:1811.06271 [gr-qc].

[16] G. Ye and Y. S. Piao, arXiv:1901.02202 [gr-qc].

[17] B. P. Abbott et al. [LIGO Scientific and Virgo Collaborations], Phys. Rev. Lett. 116, 6, 061102 (2016) [arXiv:1602.03837 [gr-qc]].

[18] B. P. Abbott et al. [LIGO Scientific and Virgo Collaborations], Phys. Rev. Lett. 119, no. 16, 161101 (2017) [arXiv:1710.05832 [gr-qc]].

[19] B. P. Abbott et al. [LIGO Scientific and Virgo Collaborations], Phys. Rev. Lett. 116, no. 22, 221101 (2016) Erratum: [Phys. Rev. Lett. 121, no. 12, 129902 (2018)] [arXiv:1602.03841 [gr-qc]].

[20] B. P. Abbott et al. [LIGO Scientific and Virgo Collaborations], arXiv:1811.00364 [gr-qc].

[21] N. Arkani-Hamed, H. C. Cheng, M. A. Luty and S. Mukohyama, JHEP 0405, 074 (2004) [hep-th/0312099].

[22] C. de Rham and S. Melville, Phys. Rev. D 95, no. 12, 123523 (2017) [arXiv:1703.00025 [hepth]].

[23] C. de Rham and S. Melville, Phys. Rev. Lett. 121, no. 22, 221101 (2018) [arXiv:1806.09417 [hep-th]].

[24] P. Creminelli, M. A. Luty, A. Nicolis and L. Senatore, JHEP 0612, 080 (2006) [hepth/0606090].

[25] M. z. Li, B. Feng and X. m. Zhang, JCAP 0512, 002 (2005) [hep-ph/0503268].

[26] E. I. Buchbinder, J. Khoury and B. A. Ovrut, Phys. Rev. D 76, 123503 (2007) [hepth/0702154].

[27] D. Langlois and K. Noui, JCAP 1602, no. 02, 034 (2016) [arXiv:1510.06930 [gr-qc]].

[28] D. Langlois, M. Mancarella, K. Noui and F. Vernizzi, JCAP 1705, no. 05, 033 (2017) [arXiv:1703.03797 [hep-th]]. 
[29] J. Gleyzes, D. Langlois, F. Piazza and F. Vernizzi, Phys. Rev. Lett. 114, no. 21, 211101 (2015) [arXiv:1404.6495 [hep-th]].

[30] H. Motohashi, K. Noui, T. Suyama, M. Yamaguchi and D. Langlois, JCAP 1607, no. 07, 033 (2016) [arXiv:1603.09355 [hep-th]].

[31] H. Motohashi and T. Suyama, Phys. Rev. D 91, no. 8, 085009 (2015) [arXiv:1411.3721 [physics.class-ph]].

[32] D. Langlois, R. Saito, D. Yamauchi and K. Noui, Phys. Rev. D 97, no. 6, 061501 (2018) [arXiv:1711.07403 [gr-qc]].

[33] J. Ben Achour, D. Langlois and K. Noui, Phys. Rev. D 93, no. 12, 124005 (2016) doi:10.1103/PhysRevD.93.124005 [arXiv:1602.08398 [gr-qc]].

[34] Paolo Creminelli, Filippo Vernizzi, Phys. Rev. Lett. 119, 251302 (2017), [arXiv:1710.05877 [astro-ph.CO]].

[35] R. Kolevatov and S. Mironov, Phys. Rev. D 94, no. 12, 123516 (2016) [arXiv:1607.04099 [hep-th]].

[36] S. Akama and T. Kobayashi, Phys. Rev. D 95, no. 6, 064011 (2017) [arXiv:1701.02926 [hepth]], S. Akama and T. Kobayashi, arXiv:1810.01863 [gr-qc].

[37] A. Ijjas, JCAP 1802 (2018) no.02, 007, [arXiv:1710.05990 [gr-qc]].

[38] S. Banerjee, Y. F. Cai and E. N. Saridakis, arXiv:1808.01170 [gr-qc].

[39] T. Kobayashi, arXiv:1901.07183 [gr-qc].

[40] J. Khoury, B. A. Ovrut, P. J. Steinhardt and N. Turok, Phys. Rev. D 64, 123522 (2001) [hep-th/0103239], J. L. Lehners, P. McFadden, N. Turok and P. J. Steinhardt, Phys. Rev. D 76, 103501 (2007) [hep-th/0702153 [HEP-TH]].

[41] Y. S. Piao, B. Feng and X. m. Zhang, Phys. Rev. D 69, 103520 (2004) [hep-th/0310206], Y. S. Piao, Phys. Rev. D 71, 087301 (2005) [astro-ph/0502343], Z. G. Liu, Z. K. Guo and Y. S. Piao, Phys. Rev. D 88, 063539 (2013) [arXiv:1304.6527 [astro-ph.CO]].

[42] Y. S. Piao, Phys. Rev. D 70, 101302 (2004) [hep-th/0407258].

[43] T. Qiu, J. Evslin, Y. F. Cai, M. Li and X. Zhang, JCAP 1110, 036 (2011) [arXiv:1108.0593 [hep-th]], T. Qiu and Y. T. Wang, JHEP 1504 (2015) 130 [arXiv:1501.03568 [astro-ph.CO]],

[44] D. Pirtskhalava, L. Santoni, E. Trincherini and F. Vernizzi, JCAP 1509, no. 09, 007 (2015) doi:10.1088/1475-7516/2015/09/007 [arXiv:1505.00007 [hep-th]].

[45] L. Santoni, E. Trincherini and L. G. Trombetta, JHEP 1808, 118 (2018) 
doi:10.1007/JHEP08(2018)118 [arXiv:1806.10073 [hep-th]].

[46] S. Mironov, V. Rubakov and V. Volkova, EPJ Web Conf. 191, 07014 (2018) [arXiv:1811.05832 [hep-th]].

[47] G. Franciolini, L. Hui, R. Penco, L. Santoni and E. Trincherini, arXiv:1811.05481 [hep-th].

[48] S. Mironov, V. Rubakov and V. Volkova, arXiv:1812.07022 [hep-th].

[49] A. Ijjas, F. Pretorius and P. J. Steinhardt, JCAP 1901, 015 (2019) [arXiv:1809.07010 [gr-qc]]. 